\title{
Magnetic resonance elastography of a uterine fibroid with massive lymphocytic infiltration — an extremely rare finding

\author{
Bogdan Obrzut ${ }^{1}\left(\mathbb{D}\right.$, Marzanna Obrzut $^{2}$, Tomasz Wasyluk' ${ }^{1}$, \\ Marcin Zmuda ${ }^{3}$, Dorota Darmochwal-Kolarz ${ }^{1}$ (D) \\ ${ }^{1}$ Department of Obstetrics and Gynecology, Institute of Medical Sciences, Medical College, University of Rzeszow, Poland \\ ${ }^{2}$ Department of Biophysics, Faculty of Mathematics and Natural Sciences, University of Rzeszow, Poland \\ ${ }^{3}$ Department of Pathomorphology, Provincial Clinical Hospital No. 2, Rzeszow, Poland
}

\section{INTRODUCTION}

Magnetic resonance elastography (MRE) is a promising tool in pre-treatment diagnostics of uterine tumors. Massive lymphocyte infiltration within a fibroid is an extremely rare finding and its elastogram has not yet been demonstrated.

\section{Case}

A 31-year-old woman, nulliparous, with no miscarriage in her medical history, was admitted for surgical treatment because of a uterine myoma. The patient had been suffering from excessive menstrual bleeding for over 12 months. A pelvic ultrasonography confirmed a heterogenous intramural tumor of the uterus, approximately $50 \mathrm{~mm}$ in diameter. A $1.5 \mathrm{~T}$ MR scanner (Optima, GE Healthcare) was used to perform the experimental MRE examination. The research protocol was approved by the Bioethics Committee of the University of Rzeszów, Poland (Reg. No. 19/04/2016). The MRE system includes special acquisition and processing software, as well as hardware consisting of an active and passive driver. The passive driver is a small plastic device that was placed over the uterus to transmit mechanical vibra-

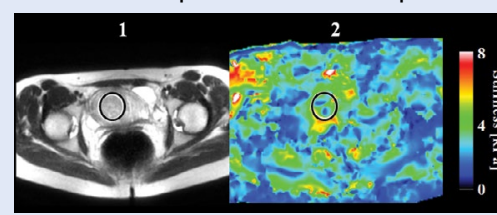

Figure 1. T2w MR image of leiomyoma with massive lymphoid infiltration (1) and its elastogram (2)

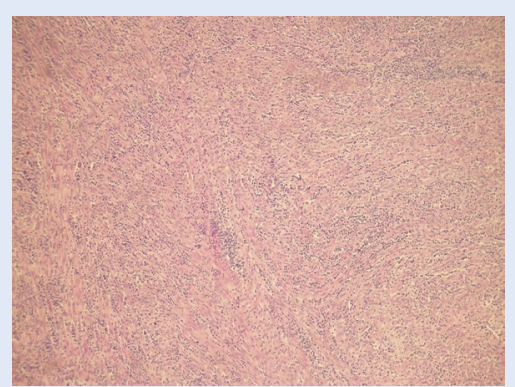

Figure 2. Leiomyoma infiltrated by immune cells. HE

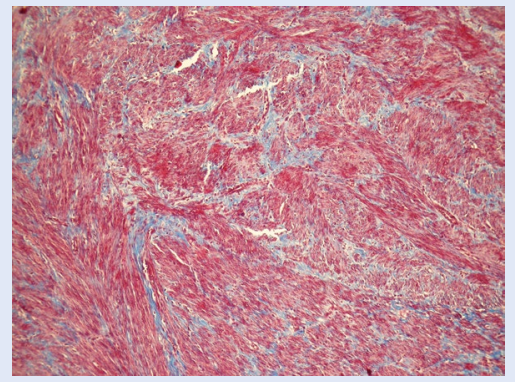

Figure 3. Trichrome Stain: fibrous tissue content less than $5 \%$ tions into the tissue. The passive driver is connected via flexible tubing to the active driver, which generates pressure pulses (at $60 \mathrm{~Hz}$ frequency). The MRE acquisition was performed using a 16-channel abdominal phase array coil. Shear wave imaging was conducted using a modified 2D gradient-recalled echo-based pulse sequence. The resulting wave images were then automatically processed to generate quantitative images depicting tissue stiffness (elastograms). Region of interest (ROI) were drawn manually on the uterine fibroid, using corresponding $\mathrm{T} 2$ images as a guide. From the $\mathrm{ROI}$, mean stiffness $(\mathrm{kPa})$ and standard deviation were reported. Finally, a laparotomy was performed on the patient and the uterine tumor was excised. The last menstrual bleeding appeared 10 days before the surgery.

\section{RESULTS}

The median stiffness of the uterine tumor was $3.36 \pm 1.21 \mathrm{kPa}$ (Fig. 1). Macroscopically, it had uterine smooth muscle wall consistency. A microscopic examination revealed leiomyoma infiltrated by lymphocytes, few scattered plasma cells, eosinophils and mast cells (Fig. 2). The concentration of extracellular components in the tumor was less than $5 \%$ (Fig. 3). Upon immunohistochemistry, in the lymphocytic infiltrate the T mature lymphocytes (CD3+/CD5+/ /TdT-) prevailed, whereas B lymphocytes (CD20+/CD5-/TdT-) were innumerous and present in nodular aggregates. The final diagnosis: leiomyoma with massive lymphoid infiltration.

\section{DISCUSSION AND CONCLUSIONS}

Massive lymphocytic infiltration in a myoma is an unusual feature. The recognition of its distinct histological features is paramount in avoiding a possible misdiagnosis. Some of them raise a strong suspicion of malignancy, especially lymphoma. The leading treatment method for uterine tumors is surgical excision allowing for their histopathological evaluation. Since most surgical techniques involve different types of morcellation, there is a risk of spreading undiagnosed neoplastic tissue within the operating field, which can negatively impact the patient's prognosis [1]. Hence, there is an urgent need for improvement of the preoperative differential diagnostics of uterine tumors, to enable the choice of the optimal treatment option. Magnetic resonance elastography offers an interesting new possibility. Malignant transformation of the tumor usually alters its mechanical property, which can be detected by MRE and potentially used in the differential diagnostics. The mean stiffness value of common leiomyomas is about $4.81 \mathrm{kPa}$ [2], depending on the concentration of the fibrotic tissue. Uterine sarcomas tend to be more "celullar", which may probably refer to other "unusual" types of myomas, also with premalignant potential [3]. The concentration of extracellular components in the presented leiomyoma with massive lymphoid infiltration was less than $5 \%$ and translated into a relatively low stiffness value of $3.36 \mathrm{kPa}$. This confirms the hypothesis, that the mechanical property of the uterine tumor may be an element of its preparative evaluation and supports the potential role of MRE in pre-treatment diagnostic algorithms.

\section{REFERRENCES}

1. George S, Barysauskas C, Serrano C, et al. Retrospective cohort study evaluating the impact of intraperitoneal morcellation on outcomes of

Corresponding author:

Tomasz Wasyluk

Department of Obstetrics and Gynecology, Institute of Medical Sciences, Medical College, University of Rzeszow, Poland

e-mail:wasylukt@gmail.com localized uterine leiomyosarcoma. Cancer. 2014; 120(20): 3154-3158, doi: 10.1002/cncr.28844, indexed in Pubmed: 24923260.

2. Jondal DE, Wang J, Chen J, et al. Uterine fibroids: correlations between MRI appearance and stiffness via magnetic resonance elastography. Abdom Radiol (NY). 2018; 43(6): 1456-1463, doi: 10.1007/s00261-0171314-1, indexed in Pubmed: 28952003.

3. Giuntoli RL, Gostout BS, DiMarco CS, et al. Diagnostic criteria for uterine smooth muscle tumors: leiomyoma variants associated with malignant behavior. J Reprod Med. 2007; 52(11): 1001-1010, indexed in Pubmed: 18161397. 